\title{
Experience of Developing a Cognitive Exercise Program in Patients Screened for Breast Cancer - A Pilot Study
}

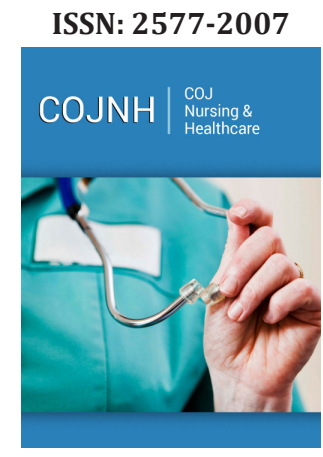

*Corresponding author: Department of Oncology-Pathology, Sarcoma and Endocrine Tumors, Karolinska Institutet, Stockholm, Sweden

Submission:

Published: 湆 November 09, 2020

Volume 6 - Issue 5

How to cite this article: Anna Schedin, Aina Johnsson, Elham Hedayati. Experience of Developing a Cognitive Exercise Program in Patients Screened for Breast Cancer - A Pilot Study. COJ Nurse Healthcare. 6(5). COJNH. 000648. 2020. DOI: $10.31031 /$ COJNH.2020.06.000648

Copyright@ Elham Hedayati, This article is distributed under the terms of the Creative Commons Attribution 4.0 International License, which permits unrestricted use and redistribution provided that the original author and source are credited.

\author{
Anna Schedin ${ }^{1}$, Aina Johnsson ${ }^{1,2,3}$ and Elham Hedayati ${ }^{3,4 *}$ \\ ${ }^{1}$ Clinic of Oncology, South Hospital, Stockholm, Sweden
}

${ }^{2}$ Neurobiology, Care Science and Society, Division of Family Medicine, Karolinska Institutet, Huddinge, Sweden

${ }^{3}$ Department of Oncology-Pathology, Karolinska Institutet, Stockholm, Sweden

${ }^{4}$ Medical Unit of Breast Cancer, Sarcoma and Endocrine Tumors, Theme Cancer, Karolinska University Hospital, Stockholm, Sweden

\begin{abstract}
Introduction: The pathophysiology for cognitive impairment is observed both in healthy women having false-positive mammography screening results and as a side-effect of breast cancer treatment. This randomized controlled pilot trial, a sub-study of Onk-kong trial aimed to compare the feasibility and acceptability of CRT versus standard of care on patients with an objective cognitive impairment in association with false-positive screening results as well as breast cancer treatment.
\end{abstract}

Method: Women with false-positive screening results or breast cancer completed the Headminder Web-based neuropsychological battery Cognitive Stability Index for response speed, processing speed, memory, and attention before diagnosis, 9 months after diagnosis (T1), and after another 3 months of follow-up (T2). A total of 31 women with an objective cognitive impairment at T1 were prospectively enrolled and randomized between training programs and expectancy. A modified psychological training program developed for schizophrenic patients, Cognitive Remediation Therapy was used and tested, by trained nurses.

Result: In total 14 women were randomized to Cognitive Remediation Therapy and 17 to expectancy. Of the randomized women, 12 fulfilled the criteria for inclusion in this study. Of these 12 women, 6 attended and fulfilled Cognitive Remediation Therapy. Five declined participation in the program, because of not having time, one couldn't start due to lack of available trainer. At T2 the results showed no improvement in any group. The dependence of the clinical setting caused problem.

Conclusion: Our study design did not enable us to demonstrate the feasibility and acceptability of the modified CRT-program among women with a breast cancer diagnosis as well as false-positive screening results or breast cancer. The study has identified several logistical issues. Changes need to be made in the conduct of the CRT to be useful also for breast cancer patients

\section{Introduction}

Breast cancer is the most frequent form of female cancer with a threefold increase between1980-2010. The present global annual incidence is 2.4 million. Survival has substantially increased over the last decades, with over $70 \%$ of women surviving at least ten years following diagnosis in countries with advanced medical care Global Burden of Disease Cancer [1]. This improved prognosis is mainly due to screening strategies that detect breast cancer in an earlier stage, and more effective multimodal treatment regimens including surgery, radio- and systemic therapies that reduce the risk of dying with about $50 \%$ [2]. Abnormal mammographic findings at screening need to be further assessed. The women are recalled for additional imaging and eventually needle biopsy to confirm whether if the radiological abnormality is benign or malign. A false-positive screening mammogram may cause distress and anxiety to the women Roman Hofvind [2].

Earlier disease detection and advances in treatment resulted in improved long-term survival in breast cancer patients and with increasing survival rates, there are a lot of breast cancer survivors that may continue with cognitive impairment with a negative impact on their quality of life and daily activities EBCTCG [3]. Objective cognitive impairment is defined as a declined score change over time on neurocognitive tests or an individual's test score that is lower compared with changes in a control population Joly [4]. The pathophysiology for cognitive impairment observed both in healthy women having false-positive mammography screening results and breast cancer treatment is still unclear. However, it is suggested 
to be multifactorial and is likely related to an interaction with sociodemographic, lifestyle, psychological, physiological, and genetic factors [5-8]. Previous studies have shown that both short and long term psychological distress are significantly higher for women having a false-positive screening mammogram such as anxiety compared to women with normal screening results $[9,10]$.

A number of studies report inconsistencies in the result that about one-third of breast cancer survivors show cognitive impairment after receiving adjuvant systemic treatments such as chemotherapy [11,12]. A meta-analysis reported that previously chemotherapy-treated breast cancer patients, observed a small magnitude of cognitive deficits [13-15]. A very subtle cognitive dysfunction, irrespective of chemotherapy, may encounter in breast cancer patients and part of which is mediated by cancerrelated post-traumatic stress [16]. There are few studies showing impairment after endocrine treatment as well $[17,18]$. Several studies have shown that even cancer treatments that are not targeted to the brain area can cause cognitive impairments e.g. radiation therapy $[18,19]$. This premise is supported by imaging studies, which reveal anatomical changes after chemotherapy as well as changes in patterns of neural activation while performing cognitive tasks [4]. There is a lack of established treatment of cognitive impairment suitable for both healthy women having false-positive mammography screening results and breast cancer patients and the current standard of care consist of wait and watch $[5,20]$. To find an optimal intervention method to improve cognitive dysfunctions is an emerging area of research in oncology [4].

There has been an interest in developing a non-medical intervention for relieving cognitive symptoms. Ferguson et al developed a program with Cognitive Behaviour Therapy directed at the memory and attention training and studied the effect on breast cancer survivors who complained of cognitive dysfunction after chemotherapy. They showed improved quality of life but no significant change in subjective cognitive function [21]. Cognitive Behaviour Therapy also showed promising results in reducing functional impairment and fatigue in cancer survivors [22]. Medical qigong training improved cancer patients' subjective reports of cognitive function and lowered inflammation markers [23]. Manualized individual interventions and group interventions have shown promise in improving neuropsychological test scores on memory, executive function, and self-report of cognitive problems and interference with daily activities [24].

Cognitive Remediation Therapy (CRT), a training program, is a set of therapies developed for improving cognitive functions in patients with schizophrenia. CRT has originally been developed and used on patients with a diagnosis of schizophrenia, but recently it has been used on cognitive rehabilitation of cognitive impairment of other diagnoses, such as brain tumor surgery, childhood psychiatric disorders, and eating disorders [5-8]. It is based on rehabilitation programs for acquired brain damages and developed in Australia and Great Britain over the last 15 years. In contrast to Cognitive Behavior Therapy, the teaching material is not personalized [25]. CRT is based on two principles, to train the impaired cognitive functions, and teach metacognition. It teaches thinking skills and cognitive strategies to compensate the cognitive impairments. The goal of the training program is to enhance the capacity and efficacy of the impaired cognitive functions, to teach broad, generalizable cognitive schemes and to enhance meta-cognition and motivation [25].

CRT has not been studied on patients with objective cognitive impairment due to false-positive screening results, breast cancer treatment, or any other cancer diagnosis, except brain tumor. An objective cognitive impairment due to false-positive screening results as well as breast cancer treatment affects the same cognitive domains as in patients with schizophrenia but in different magnitude. Thus, even when neuropsychological performance measures are subtle, breast cancer patients may be using increased cognitive resources due to reduced cognitive reserve [4]. CRT aims to improve cognitive strategies to compensate for the cognitive impairments; thus we hypothesized that CRT could be used as a cognitive remediation therapy on patients with objective cognitive impairment due to false-positive screening results or to breast cancer treatment (Figure 1).

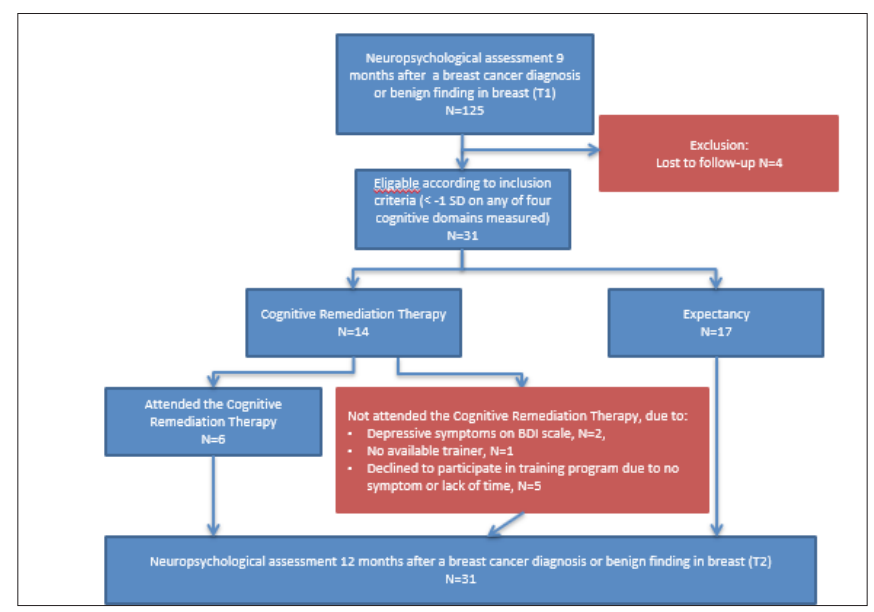

Figure 1: Disposition of 125 women in a test of cognitive function 9 months after the breast cancer diagnosis or benign was ruled out or after sector resection or mastectomy, after 6 months or after adjuvant treatment and again after additional 3 months follow-up. 
In a longitudinal study, the Onk-kong trial, we measured the effects of false-positive screening results and breast cancer treatment on cognitive functions. The Onk-kong trial and selection process has been detailed elsewhere [6,7]. This randomized controlled pilot trial, a sub-study of Onk-kong trial aimed to compare the feasibility and acceptability of CRT versus standard of care on patients with an objective cognitive impairment in association with false-positive screening results as well as breast cancer treatment.

\section{Methods}

\section{Patients}

The women with a positive radiographic finding were recruited from the prospective Onk-kong trial, between September 25, 2006, and March 2, 2009. The participants were either diagnosed with early breast cancer or a benign finding with false-positive screening results; the selection process of Onk-kong trial has been detailed elsewhere [6,7]. The participants eligible for this pilot study had to have an objective cognitive impairment nine months after breast cancer or benign diagnosis, compared to their results at enrolment in the Onk-kong trial, and randomized either to CRT training group or expectancy group. Those with depressive symptoms according to the Beck Depression Index were excluded and referred to generalpractitioners for further evaluation and treatment.

\section{Study design}

The participants completed a neuropsychological assessment nine months after breast cancer or a false-positive mammography screening results (benign diagnosis) (T1) and after another 3 months of follow-up (T2). Depression, anxiety, and quality-oflife were assessed at both test sessions, using standardized and validated questionnaires in Swedish [26,27]. The participants with cognitive impairment at $\mathrm{T} 1$ were randomized between a modified version of the CRT training or expectancy, which means the standard of care. The randomization was made in blocks of six and stratified between mild and severe cognitive impairment. The participants who were randomized to the training program were approached by telephone and asked to participate. The training program started about 4 weeks after enrolment. The participants who attended the CRT training program completed the last neuropsychological assessment (T2) one week after the last training sessions. The expectancy group performed the last neuropsychological assessment (T2) as a protocol. The participants who attended the CRT training program were asked about their experience of the training program at $\mathrm{T} 2$.

The feasibility and acceptability of CRT were measured by

i) Assessing the recruitment of the participants to the study,

ii) Assessing participant adherence to CRT, and

iii) Participant retention on CRT.

\section{Cognitive testing}

The cognitive functions were assessed using the Headminder Web-based test Cognitive Stability Index designed for repeated measurements and consists of a series of ten neurocognitive subtests which summarize in four cognitive domains; processing speed, reaction speed, attention, and memory [28]. These four domains have been validated against several traditional neuropsychological tests and have reliable psychometric properties [28].

\section{Cognitive impairment}

In order to find participants with cognitive impairment in any of the four areas of cognitive function at T1, we used a Reliable Change Index, RCI, when analyzing the results. The reliable change index is a statistical method to evaluate if the change in an individual's test performance is greater than would be expected by chance or other factors than active intervention [29-32]. The observed test result is compared with the predicted test result calculated from earlier results, divided by the standard error of prediction. The change in an individual's test score is compared with changes in a control population. It is calculated by subtracting the test score of the first assessment from the recent assessment and dividing the difference with the standard error of difference (SEdiff) in the control population. $\mathrm{RCI}=\mathrm{X} 2-\mathrm{X} 1 / \mathrm{SE}$ diff. The standard error of difference (SEdiff) was calculated from the Onk-kong trial.

Cognitive impairment was defined as if any of the four measured cognitive function domains at T1 was impaired more than $1 \mathrm{SD}$ or lower than the corresponding results of the testing session at enrolment in Onk-kong trial. If the results were lower than -1.55 SD it was considered a severe cognitive impairment. Cognitive Remediation Therapy the training program applied is based on Cognitive Remediation Therapy as presented by Delahunty et al. [33]. The training program is an individual treatment consisting of paper and pen exercises given by a therapist certified to perform CRT. It uses well-known, empirically tested learning methods, such as error-less learning, scaffolding, verbal instructions, and minimizing goals to sub-goals, for training the impaired functions $[30,31]$. The original training program consists of 40 sessions, 50 minutes each, given 3-5 days a week over a 10-12 weeks period.

We modified the CRT-program, to be shorter and at a higher cognitive level, since the cognitive impairment after false-positive screening results and breast cancer treatment was assumed to be less severe than in patients with a diagnosis of schizophrenia. The modified training program was an abbreviated version of CRT consisting of 16-18 sessions, 50 minutes each, given over 8-12 weeks. The sessions used were not altered in any way, but the more complicated sessions were selected for more efficient training in this cohort of participants with a higher cognitive level compared with schizophrenic patients. The work with modifying the program and choosing the sessions used was done under supervision of Dr. Håkan Nyman, one of the certified psychologists who introduced CRT in Sweden. Dr. Nyman has more than 30 years of experience in neuropsychology. The sessions consisted of paperand-pen-exercises and exercises with forms and symbols and hand movements. The sessions were chosen from the Swedish version of CRT, from modules Cognitive Flexibility (session 1,7,8), Memory A (session 9,11,15), Memory B (session 17-19, 22-24), Planning 
A (session 25,27,30,32,34-36) and Planning B (session 37-39) Delahunty et al. [30]. The therapists consisted of two nurses and one occupational therapist, who had participated in education on CRT to get their certification. They were recruited from an ongoing psychiatric study, where they participated as a therapist in CRT. They worked in psychiatric clinics and had earlier experience of using CRT in schizophrenic patients.

\section{Statistics}

The aim of this pilot study was to investigate the feasibility and acceptability of CRT on patients with an objective cognitive impairment in association with false-positive screening results and breast cancer treatment. The study was not designed to assess the efficacy of CRT; thus, pre-study sample size calculation was not performed. The feasibility parameters were assessing the recruitment of participants to the study, assessing participant adherence to CRT, and participant retention on CRT. All baseline characteristics are presented as means and standard deviations or medians and interquartile ranges as appropriate, frequencies, and percentages for continuous and categorical data, respectively, unless otherwise mentioned. Baseline characteristics and cognitivedomain-specific raw scores were compared between groups with independent two-tailed t-tests and $\mathrm{x}^{2}$ tests, for continuous and categorical variables, respectively. All data conformed to the assumptions of the statistical tests used to analyze them. A twosided 5\% critical level for a statistical significance was employed. Statistical analyses were performed using SAS version 9.2 for Windows and SPSS version 18 for Mac OS.

\section{Ethical Consent}

The Regional Ethics Review Board at Karolinska Institute, in Stockholm, approved this study, and all participants gave their written informed consent before enrolment (Dnr 2006/560-31/2).

\section{Result}

Of the 68 with newly diagnosed early breast cancer and 57 benign finding women who completed a neuropsychological assessment at T1, 31 had cognitive impairment, 14 were randomized to attend the CRT training program and 17 were randomized to expectancy. Six of the 14 participants randomized to training program attended and fulfilled the program; two were excluded due to depressive symptoms on the BDI-scale and one couldn't start the program due to problems with finding an available trainer at the time; Five declined participation in the program, two because of having no subjective problems with cognition, three because of not having time. The participants randomized to the training program, who didn't attend the program, were grouped together with the participants randomized to expectancy, forming the expectancy group. The participants who attended the training program formed the training group. The training group consisted of 5 participants with breast cancer and 1 participant with benign findings. The expectancy group consisted of 14 participants with breast cancer and 11 participants with benign finding. The training group was two years older, was less married/cohabiting, and worked less part-time than the expectancy group. The level of education was similarly distributed in both groups (Table 1).

Table 1: Baseline demographic and characteristics of 31 women with cognitive impairment 9 months after breast cancer or benign diagnosis, randomized to training or expectancy.

\begin{tabular}{|c|c|c|c|}
\hline Characteristic & Training $(\mathrm{N}=6)$ & Expectancy $(\mathrm{N}=25)$ & Total $(\mathrm{N}=31)$ \\
\hline Age mean (SD mean), years & $59,6(6,9)$ & $58,2(8,0)$ & $58,5(7,7)$ \\
\hline Age categories, $40-49$ years & 0 & 3 & 3 \\
\hline Age categories, $50-59$ years & 2 & 10 & 12 \\
\hline Age categories, $60-70$ years & 4 & 12 & 16 \\
\hline Education $<12$ years & 3 & 14 & 17 \\
\hline Education $>13$ years & 3 & 11 & 14 \\
\hline Civil status Married/ Cohabiting & 3 & 18 & 21 \\
\hline Civil status Single/ Divorced/ Widowed & 3 & 7 & 10 \\
\hline Work status Full time & 2 & 7 & 9 \\
\hline Work status Part time & 0 & 6 & 6 \\
\hline Work status Unemployed & 4 & 12 & 16 \\
\hline Breast cancer & 5 & 14 & 19 \\
\hline Benign finding & 1 & 11 & 12 \\
\hline Menopausal status Premenopausal & 0 & 7 & 7 \\
\hline Menopausal status Peri- or postmenopausal & 6 & 18 & 24 \\
\hline CSI, Processing speed, mean (SD-mean) & $4.0(0.9)$ & $3.9(0.9)$ & $3.9(0.9)$ \\
\hline CSI, Response speed, mean (SD-mean) & $0.8(0.2)$ & $0.7(0.1)$ & $0.7(0.1)$ \\
\hline CSI, Attention, mean (SD-mean) & $11.0(3.7)$ & $12.0(3.8)$ & $11.8(3.8)$ \\
\hline
\end{tabular}




\begin{tabular}{|c|c|c|c|}
\hline CSI, Memory, mean (SD-mean) & $7.8(0.4)$ & $7.3(1.2)$ & $7.4(1.1)$ \\
\hline Beck Anxiety Inventory & $13.3(7.6)$ & $10.0(9.6)$ & $10.6(9.2)$ \\
\hline Beck Depression Inventory & $13.2(7.8)$ & $8.2(9.8)$ & $9.1(9.6)$ \\
\hline Global Health Status & $62.5(20.2)$ & $70.3(26.4)$ & $68.8(25.2)$ \\
\hline
\end{tabular}

\section{Cognitive functions}

The results showed no significant change in results between $\mathrm{T} 1$ and T2 in any of the groups (Table 2). Processing speed was lower in the training group $(-0,33 \mathrm{SD})$ than in the expectancy group $(0,68$
SD). The training group had higher results in response speed and attention than the expectancy group $(0,79$ SD respectively $0,49 \mathrm{SD}$ and 0,50 SD respectively $-0,03 \mathrm{SD}$ ). In both groups the results in memory were equal $(0,27 \mathrm{SD}$ respectively $0,28 \mathrm{SD})$.

Table 2: Change in test scores from the four areas of cognitive function among 31 women with cognitive impairment at 9 months after breast cancer or benign diagnosis compared to 12 months after breast cancer or benign diagnosis, randomized to training or expectancy.

\begin{tabular}{|c|c|c|c|}
\hline \multirow{2}{*}{ Cognitive Function } & Training & Expectancy & N=25 \\
\cline { 2 - 4 } & $\mathbf{N = 6}$ & $0.68(-0.44-1.79)$ & 0.34 \\
\hline Performance speed, Mean $(95 \% \mathrm{CI})$ & $-0.33(-1.09-0.44)$ & $0.49(-0.43-1.41)$ & 0.75 \\
\hline Response speed, Mean $(95 \% \mathrm{CI})$ & $0.79(-0.31-1.90)$ & $-0.03(-0.65-0.58)$ & 0.41 \\
\hline Attention, Mean $(95 \% \mathrm{CI})$ & $0.50(-0.19-1.18)$ & $0.28(-0.31-0.88)$ & 0.99 \\
\hline Memory, Mean $(95 \% \mathrm{CI})$ & $0.27(-1.23-1.77)$ & \\
\hline
\end{tabular}

\section{Feasibility of cognitive remediation therapy training program}

CRT has been successfully used to improve the function of impaired cognitive ability Delahunty et al. [30]. We aimed to study if CRT was also acceptable in the context that is the everyday life of breast cancer patients. Recruitment of randomized participants in the modified training program of CRT was demanding since five participants declined to attend due to lack of time. The participants attending the program made an effort and spend a lot of time fulfilling the program. All six participants attended the whole program, in total 16 sessions of 50 minutes each, during a period of 8-12 weeks. For those with breast cancer, it was not possible to synchronize their appointment time for CRT with their clinical routine follow-up regime for breast cancer survivors as part of their cancer survivorship care plan, due to lack of resource flexibility. Four of the participants attending the training program gave their view of the training program afterward. They all thought the program was a positive experience. One thought it took too long time and one thought it was too easy. There were three different therapists involved in the study since the recruitment period lasted a long time and they had other engagements as well. They supervised two participants each. The therapist could only supervise one participant at the time in the training program due to the time consumption. At one time there was no therapist available which lead to that one participant was not able to start the training program.

\section{Discussion}

This small pilot study demonstrates the feasibility and acceptability of CRT, a modified cognitive training program, on a group of women with an objective cognitive impairment in association with false-positive screening results or breast cancer treatment. In addition, the study provides an opportunity to test the process of recruiting participants, adherence to CRT, and retention of participants on CRT. Moreover, the study provides possible advice for the continued development of cognitive training programs such as a modified version of Cognitive Remediation Therapy. Our study design did not enable us to demonstrate the feasibility and acceptability of the modified CRT program among patients with an objective cognitive impairment associated with false-positive screening results and breast cancer treatment. All the eligible patients accepted to participate in the study, thus recruitment of participants to the study was high. The modified CRT-program contained too many sessions over time that is why we needed to involve three different therapists. The therapists were not solely dedicated to this pilot project, leading to less participant adherence to CRT.

Moreover, the participants randomized to CRT perceived the program as time-consuming and some could not accommodate the scheduled CRT-program, leading to dropouts and low participant retention on CRT Five out of 11 participants randomized to CRT stated that they perceived the program as time- consuming and preferred not to attend. However, the adherence and retention of participants on CRT were good since all six participants who began the program completed it. It was not possible to combine the training sessions with clinical routine follow-up regime for breast cancer survivors as part of their care plan since the CRT was time-consuming and the routine health care system lacks flexibility. The challenge was to find an intervention that suited the patient population and offered cognitive rehabilitation. CRT as developed by Delahunty et al. [30] has successfully been used in studies of patients with cognitive impairment due to a diagnosis 
of schizophrenia Delahunty et al. [30]. Meta-analysis shows a small to moderate effect of cognitive rehabilitation on (global) cognitive outcome Delahunty et al. [30]. CRT has been used on cognitive rehabilitation of cognitive impairment of other diagnoses, such as brain tumor surgery, childhood psychiatric disorders, and eating disorders. Cancer-related cognitive impairment can affect cognitive abilities including attention, memory, executive functions, and processing speed [33]. Equivalent cognitive abilities are affected in patients with schizophrenia [33]. CRT is designed specially to rehabilitate attention, memory, and executive function, thus we believed it is a suitable program to be used as a cognitive remediation therapy. We needed to modify the program, to fit patients with more moderate cognitive impairment than those of patients with schizophrenia. We didn't change the content of the program, but we shortened it down. Despite that, the training program was time-consuming.

A previous study has shown that only one female cancer patient out of four desire psychological support and interventions [34]. A recent study showed similar finding, one in five patients with metastatic colorectal cancer receiving first-line palliative treatment used psychosocial services [35]. These studies are consistent with our results of only half of the women with impaired cognitive functions randomized to the training program participate, to attend in the modified training program of CRT, and making the process of recruiting participants difficult. Those participants that declined found the program time-consuming. However, we had already shortened the training program from the original program and further reduction causes problems with the effect of the program. The participants randomized to the training program, who didn't attend the program, were grouped together with the participants randomized to expectancy.

This was done in order to study if the training program in itself gave any effect on improving cognitive impairment. The lack of positive results from the training program may be due to the small sample size and the reduction of the material that we made. The training program needs certified therapists to be effective. We had problems with keeping the same therapist throughout the whole study. The problem with the lack of continuity among the therapists made the implementation of the study difficult, although it is not clarified whether it affected the patients' results. Beyond the followup assessments, we did not use any specific questionnaires or tools to further evaluate the feasibility and acceptability. The level we chose to classify and detect impairment in cognitive function, 1 SD below the individual's baseline result, was wider than normal and allowed for a greater level of chance.

We chose this level in order to be able to detect a more subtle cognitive impairment since the literature implied that cognitive dysfunction after breast cancer treatment is mild. In doing that we knew that some of the individuals that would have a result implying cognitive impairment would do so due to chance and not to a true impairment. The number of interventional studies for improving cognitive impairment after breast cancer treatment has increased in recent years but are still few [36-38]. Pharmacological intervention for fatigue and cognitive impairment after cancer treatment has been tested in small studies, mainly with central nervous system stimulants used for attention disorders, such as Modafinil and Methylphenidate. Modafinil has been shown to enhance some memory and attention skills after adjuvant treatment with chemotherapy in breast cancer survivors [39]. Methylphenidate shows no improvement in quality of life or fatigue.

In conclusion, the need for finding an intervention for rehabilitating cognitive impairment due to false-positive screening results or breast cancer treatment is still strong. We think that our modified training program from CRT is ambitious and could be worth developing further. This study has identified several logistical issues and changes need to be made in the conduct of the CRT in order to be useful also for breast cancer patients. Regardless of the training method used, clear coordination between the oncological treatment and the training program is needed; the training takes place in premises in connection with the oncological activity and is led by oncological staff such as CRT certified contact nurses if possible and adapt the training sessions so that the patients who work do not need to take time off from work. The latter is not least important as patients with false-positive results should be able to participate [40-42].

\section{Acknowledgment}

The authors acknowledge the cooperation of the research nurse Marika Hjelmqvist who coordinated this multi-professional and multi-disciplinary pilot study. We would like to acknowledge the cooperation of all the nurses in the breast cancer care team for their support and flexibility to ease the process for the participants in this study. The authors also acknowledge the cooperation of the senior statistician Hassan Alinaghizadeh at Karolinska Institute, who performed the analysis of this study.

\section{References}

1. Global Burden of Disease Cancer C (2017) Global, regional, and national cancer incidence, mortality, years of life lost, years lived with disability, and disability-adjusted life-years for 32 cancer groups, 1990 to 2015: A systematic analysis for the global burden of disease study. JAMA Oncology 3(4): 524-548.

2. EBCTCG EA (2012) Comparisons between different polychemotherapy regimens for early breast cancer: meta-analyses of long-term outcome among 100,000 women in 123 randomised trials. Lancet 4(379): $432-444$.

3. Roman M, Hofvind S, von Euler Chelpin M, Castells X (2019) Long-term risk of screen-detected and interval breast cancer after false-positive results at mammography screening: joint analysis of three national cohorts. Br J Cancer 120(2): 269-275.

4. Joly F, Giffard B, Rigal O, De Ruiter MB, Small BJ, et al. (2015) Impact of cancer and its treatments on cognitive function: Advances in research from the paris international cognition and cancer task force symposium and update since 2012. J Pain Symptom Manage 50(6): 830-841.

5. Ahles TA, Root JC (2018) Cognitive Effects of Cancer and Cancer Treatments. Annu Rev Clin Psychol 14: 425-451.

6. Hedayati E, Alinaghizadeh H, Schedin A, Nyman H, Albertsson M (2012) Effects of adjuvant treatment on cognitive function in women with 
early breast cancer. European Journal of Oncology Nursing: The official journal of European Oncology Nursing Society 16(3): 315-322.

7. Hedayati E, Schedin A, Nyman H, Alinaghizadeh H, Albertsson M (2011) The effects of breast cancer diagnosis and surgery on cognitive functions. Acta Oncol 50(7): 1027-1036.

8. Wefel Jeffrey S, Kesler Shelli R, Noll Kyle R, Schagen Sanne B (2014) Clinical characteristics, pathophysiology, and management of noncentral nervous system cancer-related cognitive impairment in adults. A Cancer Journal for Clinicians 65(2): 123-138.

9. Bond M, Pavey T, Welch K, Cooper C, Garside R, et al. (2013) Systematic review of the psychological consequences of false-positive screening mammograms. Health Technol Assess 17(13): 1-170.

10. Feng Y, Zhang XD, Zheng G, Zhang LJ (2019) Chemotherapy-induced brain changes in breast cancer survivors: evaluation with multimodality magnetic resonance imaging. Brain Imaging Behav 13(6): 1799-1814.

11. Ganz PA, Kwan L, Castellon SA, Oppenheim A, Bower JE, et al. (2013) Cognitive complaints after breast cancer treatments: examining the relationship with neuropsychological test performance. JNCI: Journal of the National Cancer Institute 105(11): 791-801.

12. Hermelink K, Buhner M, Sckopke P, Neufeld F, Kaste J, et al. (2017) Chemotherapy and Post-traumatic Stress in the Causation of Cognitive Dysfunction in Breast Cancer Patients. J Natl Cancer Inst 109(10):

13. Hermelink K, Voigt V, Kaste J, Neufeld F, Wuerstlein R, et al. (2015) Elucidating pretreatment cognitive impairment in breast cancer patients: The impact of cancer-related post-traumatic stress. JNCI: Journal of the National Cancer Institute 107(7): djv099-djv099.

14. Menning S, de Ruiter MB, Kieffer JM, Agelink van Rentergem J, Veltman DJ, et al. (2016) Cognitive impairment in a subset of breast cancer patients after systemic therapy-results from a longitudinal study. J Pain Symptom Manage 52(4): 560-569.e561.

15. Arnaboldi P, Riva S, Crico C, Pravettoni G (2017) A systematic literature review exploring the prevalence of post-traumatic stress disorder and the role played by stress and traumatic stress in breast cancer diagnosis and trajectory. Breast Cancer (Dove Med Press) 9: 473-485.

16. Hegel MT, Moore CP, Collins ED, Kearing S, Gillock KL, et al. (2006) Distress, psychiatric syndromes, and impairment of function in women with newly diagnosed breast cancer. Cancer 107(12): 2924-2931.

17. Jim HSL, Phillips KM, Chait S, Faul LA, Popa MA, et al. (2012) MetaAnalysis of cognitive functioning in breast cancer survivors previously treated with standard-dose chemotherapy. Journal of Clinical Oncology 30(29): 3578-3587.

18. Lee PE, Tierney MC, Wu W, Pritchard KI, Rochon PA (2016) Endocrine treatment-associated cognitive impairment in breast cancer survivors: evidence from published studies. Breast Cancer Res Treat 158(3): 407420.

19. Shibayama O, Yoshiuchi K, Inagaki M, Matsuoka Y, Yoshikawa E, et al. (2014) Association between adjuvant regional radiotherapy and cognitive function in breast cancer patients treated with conservation therapy. Cancer Med 3(3): 702-709.

20. Shibayama O, Yoshiuchi K, Inagaki M, Matsuoka Y, Yoshikawa E, et al. (2019) Long-term influence of adjuvant breast radiotherapy on cognitive function in breast cancer patients treated with conservation therapy. Int J Clin Oncol 24(1): 68-77.

21. Underwood E, Rochon P, Moineddin R, Lee P, Wu W, et al. (2018) Cognitive sequelae of endocrine therapy in women treated for breast cancer: a meta-analysis. Breast Cancer Research and Treatment 168(2): 299-310.
22. Fernandes HA, Richard NM, Edelstein K (2019) Cognitive rehabilitation for cancer-related cognitive dysfunction: a systematic review. Support Care Cancer 27(9): 3253-3279.

23. Ferguson RJ, McDonald BC, Rocque MA, Furstenberg CT, Horrigan S, et al (2012) Development of CBT for chemotherapy-related cognitive change: results of a waitlist control trial. Psychooncology 21(2): 176-186.

24. Gielissen MF, Verhagen S, Witjes F, Bleijenberg G (2006) Effects of cognitive behavior therapy in severely fatigued disease-free cancer patients compared with patients waiting for cognitive behavior therapy: a randomized controlled trial. J Clin Oncol 24(30): 4882-4887.

25. Oh B, Butow PN, Mullan BA, Clarke SJ, Beale PJ, et al. (2012) Effect of medical Qigong on cognitive function, quality of life, and a biomarker of inflammation in cancer patients: a randomized controlled trial. Support Care Cancer 20(6): 1235-1242.

26. Becker H, Henneghan AM, Volker DL, Mikan SQ (2017) A Pilot Study of a Cognitive-Behavioral Intervention for Breast Cancer Survivors. Oncol Nurs Forum 44(2): 255-264.

27. Wykes T, vander Gaag M (2001) Is it time to develop a new cognitive therapy for psychosis--cognitive remediation therapy (CRT)? Clin Psychol Rev 21(8): 1227-1256.

28. Wykes TCR (2005) Cognitive remediation therapy for schizophrenia. Theory and Practise ( $1^{\text {st }}$ edn), Taylor and Francis Group, Routledge, UK.

29. Aaronson NK, Ahmedzai S, Bergman B, Bullinger M, Cull A, et al. (1993) The European Organization for Research and Treatment of Cancer QLQ-C30: A Quality-of-Life Instrument for Use in International Clinical Trials in Oncology. JNCI: Journal of the National Cancer Institute 85(5): 365-376.

30. Beck AT, Epstein N, Brown G, Steer RA (1988) An inventory for measuring clinical anxiety: psychometric properties. Journal of Consulting and Clinical Psychology 56(6) 893-897.

31. Erlanger DM, Kaushik T, Broshek D, Freeman J, Feldman D, et al. (2002) Development and validation of a web-based screening tool for monitoring cognitive status. J Head Trauma Rehabil 17(5): 458-476.

32. Neil S, Jacobson LJR, Sara B, Berns Joseph B, McGlinchey (1999) Methods for defining and determining the clinical significance of treatment effects: Description, application, and alternatives. Journal of Consulting and Clinical Psychology 67(3): 300-307.

33. Delahunty A, Reeder C, Wykes T, Morice R, Newton E (2002) Revised Cognitive Remediation - Therapy Manual. Retrieved from London, UK.

34. Kern RS, Green MF, Mitchell S, Kopelowicz A, Mintz J, et al. (2005) Extensions of errorless learning for social problem-solving deficits in schizophrenia. Am J Psychiatry 162(3): 513-519.

35. Mar Fan HG, Clemons M, Xu W, Chemerynsky I, Breunis H, et al. (2008) A randomised, placebo-controlled, double-blind trial of the effects of d-methylphenidate on fatigue and cognitive dysfunction in women undergoing adjuvant chemotherapy for breast cancer. Support Care Cancer 16(6): 577-583.

36. Merckaert I, Libert Y, Messin S, Milani M, Slachmuylder JL, et al. (2010) Cancer patients' desire for psychological support: prevalence and implications for screening patients' psychological needs. Psychooncology 19(2): 141-149.

37. Schuurhuizen C, Braamse AMJ, Konings I, Verheul HMW, Dekker J (2019) Predictors for use of psychosocial services in patients with metastatic colorectal cancer receiving first line systemic treatment. BMC Cancer 19(1): 115.

38. Damholdt MF, Mehlsen M, O'Toole MS, Andreasen RK, Pedersen AD, et al. (2016) Web-based cognitive training for breast cancer survivors with 
cognitive complaints-a randomized controlled trial. Psychooncology 25(11) 1293-1300.

39. Park JH, Jung YS, Kim KS, Bae SH (2017) Effects of compensatory cognitive training intervention for breast cancer patients undergoing chemotherapy: A pilot study. Support Care Cancer 25(6): 1887-1896.

40. Swainston J, Derakshan N (2018) Training cognitive control to reduce emotional vulnerability in breast cancer. Psychooncology 27(7): 17801786.
41. Kohli S, Fisher SG, Tra Y, Adams MJ, Mapstone ME, et al. (2009) The effect of modafinil on cognitive function in breast cancer survivors. Cancer 115(12): 2605-2616.

42. Salz T, Richman AR, Brewer NT (2010) Meta-analyses of the effect of false-positive mammograms on generic and specific psychosocial outcomes. Psychooncology 19(10): 1026-1034.

For possible submissions Click below: 